\title{
PERFIL DA FAUNA DE VESPAS PARASITÓIDES (INSECTA, HYMENOPTERA) EM UMA ÁREA DE MATA ATLÂNTICA DO PARQUE ESTADUAL DE PEDRA AZUL, DOMINGOS MARTINS, ESPÍRITO SANTO, BRASIL
}

\section{I.D.C.C. Alencar, F.B. Fraga, M.T. Tavares, C.O . Azevedo}

Universidade Federal do Espírito Santo, Departamento de Ciências Biológicas, Av. Marechal Campos, 1468, CEP 29040-090, Vitória, ES, Brasil. E-mail: idccalencar@gmail.com

\section{RESUMO}

\begin{abstract}
Este trabal ho teve como objetivo conhecer a fauna de vespas parasitóides ocorrentes em área de Mata Atlântica no Parque Estadual de Pedra Azul, Espírito Santo, Brasil. Foram coletadas 1.997 vespas parasitói des pertencentesa 28famílias através de 16armadilhas Mal aise expostas por 14 dias. As famílias Diapriidae, Platygastridae e Bethylidae apresentaram as maiores abundâncias relativas (26,54\%, 11,47\% e10,72\%, respectivamente). Quatorzefamílias apresentaram abundância relativa inferior a 1\%. A subfamília Charipinae (Figitidae) é registrada pela primeira vez para o Estado do Espírito Santo. É apresentada lista de gêneros e espécies de algumas famílias.
\end{abstract}

PALAVRAS-CHAVE: A bundância, biodiversidade, levantamento, novos registros.

\section{ABSTRACT}

OUTLINE OF THE PARASITOID WASPS (INSECTA, HYMENOPTERA) IN AN ATLANTIC RAIN FOREST AREA FROM PARQUE ESTADUAL DE PEDRA AZUL, DOMINGOSMARTINS, ES, BRAZIL. A survey was conducted of the parasitic wasps from Parque Estadual de Pedra Azul in EspíritoSantoState, Brazil. A total of 1,997specimens of parasitoid wasps belonging to 28 families were collected with 16 Malaise traps exposed for 14 days. Diapriidae, Platygastridae and Bethylidae presented the highest relative abundance ( $26.54 \%, 11.47 \%$ and $10.72 \%$, respectively). Fourteen families presented relative abundance lower than $1 \%$. The subfamily Charipinae (Figitidae) was recorded for the first time in Espírito Santo State. A list of genera and species is presented.

KEY WORDS: A bundance, biodiversity, survey, first reports.

\section{INTRODUÇÃO}

Os Hymenoptera são abundantes e ocupam os mais diversos tipos de ambientes. A tualmente estão incluídas nesta ordem cerca de 115.000 espécies (HANSON; GAULD, 1995) e, segundo BRANDÃo (1999), a ordem éconsi derada aquartamaisespeciosaentreos Insecta; estimativas recentes indicam que esta posição pode ser subestimada.

Entre os Hymenoptera, os parasitóides têm grande importância para a manutenção do equilíbrio ambiental atuando na regulação populacional de diversos insetos hospedeiros (BORROR et al., 1992; LaSalle; Gauld, 1993).

Poucos estudos relatam a riqueza de famílias de vespas parasitóides em áreas de conservação no Estado do Espírito Santo (Azevedo; Santos, 2000; A zevedo et al., 2002; AzEVEDo et al., 2003), o que justificou a real ização desseestudo, cujo objetivo foi traçar o perfil dafaunadevespasparasitóidesdo ParqueEstadual de Pedra Azul, no Muniápio de Domingos Martins, ES.

\section{MATERIAL E MÉTODOS}

A amostragem foi realizada no Parque Estadual de Pedra Azul (PEPAZ) (2025'55"S, 41으는'W) localizadono MunicípiodeDomingosMartins, Espírito Santo. A quela unidade de conservação, de 1.240 ha é caracterizada por relevo montanhoso, com formações rochosas queal cançam $1.822 \mathrm{~m}$ deal titudee vegetação predominante de Floresta Ombrófila Altimontana, Mata Atlântica.

A amostragem seguiu os protocolos de levantamento rápido de espécies (H ERzog et al., 2002; ERftemeijer, 2002; Simonson et al., 2001; Smith et al., 1999; Stohlgren et al., 1997; DaIly; Ehrlich, 1995; Oliver; Beattie, 1993), que vêm assumindo papel 
importantenaidentificação deáreasprioritáriaspara a conservação da biodiversidade.

Os espécimes foram coletados no período de 26 de agosto a 2 desetembro de 2003, utilizando 16 armadiIhas Malaise, totalizandoesforço amostral total de224 armadilhas/ dia. As vespas parasitóides coletadas foram identificadasanível defamíliacomoauxíliodas chaves deFernánDEZ;SHARKEY (2006) eGRISSELLSChAufF (1997). N este estudo foram incluídas as famílias pertencentesàsérieParasitica(Terebrantia), aspertencentes à superfamília Chrysidoidea e as famílias de Vespoidea que apresentam hábito parasitóide. O material coletado foi depositado na Coleção Entomológica do Departamento de Ciências Biológicas daUniversidadeFederal doEspíritoSanto (UFES).

\section{RESULTADOSE DISCUSSÃO}

Foram amostrados 1.997 exemplares de vespas parasi tóides pertencentes a 28 famílias (Tabela 1 ).

A s famílias col etadas no PEPAZ são comumente capturadas comautilização dearmadilha Mal aise. $\mathrm{O}$ registro dafamíliaSclerogibbidaeéfato relevantepor ser pouco freqüente em estudos faunísticos. A ocorrência da subfamília Charipinae é registrada pela primeiravez parao Estado do EspíritoSanto, embora al gumas desuas espécies sejam bastantecomuns em áreas de horticultura.

DasnovesuperfamíliascoletadasProctotrupoidea foi a mais freqüente, com $30,15 \%$ dos exemplares coletados, seguida por Platygastroidea (18,88\%), Ichneumonoidea (16,52\%), Chalcidoidea (14,96\%), Chrysidoidea (11,02\%), Cynipoidea $(4,51 \%)$, Evanioidea (1,95\%), Vespoidea (1,10\%) e Ceraphronoidea (0,85\%).

A riqueza de famílias obtida neste estudo é semeIhanteàquela obtida para outras áreas de conservação doEstadodoEspíritoSanto.A zEVEDo etal.(2003)obtiveram 31 famílias utilizando o mesmo esforço amostral com armadilha Malaise para a Estação Biológica de Santa Lúcia. Azevedo; Santos (2000) e Azevedo et al. (2002) obtiveram 30 e28famílias paraa Reserva Biológica de Duas Bocas e para o ParqueEstadual da Fonte Grande, respectivamente, ambos utilizando varredura de vegetação ao longo de um ano. Em levantamentos rápidos com varredura da vegetação, MRS Estudos AmbientAIs (2000a, 2000b) obtiveram28e21famílias de vespasparasitóidesparaosParquesEstaduaisdeForno Grandee da Cachoeira da Fumaça, respectivamente.

EmestudosrealizadosemMataA tlântica, noEstado deSãoPaulo, utilizandoarmadilasdeM öricke, Malaise evarredura devegetação, PERIOTO;L ARA (2003) ePERIOTO et al. (2005) obtiveram 23 e 26 famílias de vespas parasitóides no Parque Estadual da Serra do Mar ena Estação Ecológica Juréia-Itatins, respectivamente.
Tabela 1 - Abundância das superfamílias e famílias de vespas parasitóides amostradas por meio de armadilha Malaise no Parque Estadual de Pedra Azul, Domingos $M$ artins, ES, no período de 26 de agosto a 2 de setembro de 2003.

\begin{tabular}{|c|c|c|}
\hline Táxon & Abundância & $\begin{array}{c}\text { Freqüência } \\
(\%)\end{array}$ \\
\hline Chrysidoidea & 220 & 11,02 \\
\hline Bethylidae & 214 & 10,72 \\
\hline Chrysididae & 1 & 0,05 \\
\hline Dryinidae & 4 & 0,20 \\
\hline Sclerogibbidae & 1 & 0,05 \\
\hline Vespoidea & 22 & 1,10 \\
\hline Mutillidae & 1 & 0,05 \\
\hline Tiphiidae & 21 & 1,05 \\
\hline Ceraphronoidea & 17 & 0,85 \\
\hline Ceraphronidae & 17 & 0,85 \\
\hline Chalcidoidea & 300 & 15,01 \\
\hline Aphelinidae & 4 & 0,20 \\
\hline Chalcididae & 1 & 0,05 \\
\hline Encyrtidae & 41 & 2,05 \\
\hline Eulophidae & 131 & 6,56 \\
\hline Eupelmidae & 8 & 0,40 \\
\hline Eurytomidae & 6 & 0,30 \\
\hline Mymaridae & 63 & 3,15 \\
\hline Pteromalidae & 42 & 2,10 \\
\hline Torymidae & 1 & 0,05 \\
\hline Trichogrammatidae & 3 & 0,15 \\
\hline Cynipoidea & 90 & 4,51 \\
\hline Figitidae & 90 & 4,51 \\
\hline Evanioidea & 39 & 1,95 \\
\hline Evaniidae & 39 & 1,95 \\
\hline Ichneumonoidea & 330 & 16,52 \\
\hline Braconidae & 166 & 8,31 \\
\hline Ichneumonidae & 164 & 8,21 \\
\hline Platygastroidea & 377 & 18,88 \\
\hline Platygastridae & 229 & 11,47 \\
\hline Scelionidae & 148 & 7,41 \\
\hline Proctotrupoidea & 602 & 30,15 \\
\hline Diapriidae & 530 & 26,54 \\
\hline Monomachidae & 71 & 3,56 \\
\hline Proctotrupidae & 1 & 0,05 \\
\hline Total & 1.997 & 100 \\
\hline
\end{tabular}

O potencial faunístico do PEPAZ para alguns gruposdeH ymenopterafoi verificado principalmente na família Bethylidae que apresentou 47 espécies coletadas, pertencentes a cinco gêneros: A penesia Westwood, 1874; D issomphalus A shmead, 1893;Epyris Westwood, 1832; Holepyris Kieffer, 1905 e 
Pseudisobrachium Kieffer, 1904. Destas, sete espécies foram identificadas: A penesiaconcavata Correa\& Azevedo, 2001, A . elongata Evans, 1963, D issomphalus krombeini (Azevedo, 1999), D . punctatus (Kieffer, 1910) D. plaumanni (Evans, 1964), D. rectilineus Azevedo, 1999, D. umbilicus A zevedo, 2003. A o menos 24 espé cies de Bethylidae são novas para a ciência e serão descritas oportunamente.

Outros gêneros também foram identificados: em Eulophidae, A prostocetus Westwood, 1833, Euderus Haliday, 1844 e Tetrastichus Haliday, 1844; em Encyrtidae, M etaphycus Mercet,1917; emEupelmidae, Brasema Cameron,1884 eE upel mus Dalman, 1820; em Eurytomidae, Eurytoma Illiger, 1807 e Sycophila Walker, 1871; emA phelinidae, Encarsia Förster, 1878. Trêsespécies deM onomachidaeforamidentificadas: M onomachus eurycephalus Schetterer, 1890, M . fuscator Perty, $1833 \mathrm{eM}$. sp. U maespécienovadeChalcididae, pertencente ao gênero Brachymeria Westwood, 1829, também foi identificada. O gênero Probethylus A shmead, 1902 (Sclerogibbidae) foi amostrado e, recentementefoi reportado também para os estados do N ortee Sudeste do Brasil, incluindo o Espírito Santo (Olmi, 2005).

Segundo o Plano de Manejo do PEPAZ (IDAF, 2004), aentomofaunado parquetemgrandepotencial para abrigar espécies raras e grande proporção de espécies novas para al guns grupos de Hymenoptera como pôdeser demonstrado neste estudo. Cabe ressaltar que, no período das campanhas de coleta, a predominância detemperaturas baixas pode ter afetado de maneira si gnificativa a amostragem.

\section{AGRADECIMENTOS}

À Maria Isabel Martins Nascimento, do Instituto deDefesaAgroflorestal doEspíritoSanto (IDA F), pela autorização das coletas naárea deestudo eao Centro de Pesquisa do $\mathrm{M}$ ar (Cepemar) pelo apoio financeiro esuportelogístico para realização das coletas edeste estudo.

REFERÊNCIAS

Azevedo, C.O; Corrêa, M.S.; GobB, F.T.;.K awada, R.; Lanes, G.O.; Moreira, A.R.; RedighierI , E.S.; SAntos, L.M.; Waichert, C. Perfil dasfamíliasdevespasparasitóides(Hymenoptera) em uma área de MataA tlântica da Estação Biológica de Santa Lúcia, Santa Teresa, ES, Brasil. Bol etim do M useu de Biologia M ello Letãão, n.16, p39-46, 2003.

Azevedo, C.O.;SAntos, H.S. Perfil dafauna dehimenópteros parasitóides (Hymenoptera) em uma área de Mata Atlântica da Reserva Biológica de Duas Bocas, Cariacica, ES, Brasil . Bol etim do M useu deBiologia M ello Leitão, n.11/ 12, p.117-126, 2000.
Azevedo, C.O.; Kawada, R.; Tavares, M.T.; Perioto, N.W. Perfil dafaunadehimenópterosparasitóides(Insecta, Hymenoptera) em uma área de Mata Atlântica o ParqueEstadual daFonteGrande, Vitória, ES, Brasil. Revista Brasileira de Entomologia, v.46, n.2, p.133-137, 2002.

Borror, D.J.; Triplehorn, C.A.; Johnson, N.F. (Eds.). A n introduction to the study of insects. 6th.ed. Orlando, Flórida: Saunders College Publishing, 1992. 875p.

BrandÃo, C.R.F. Hymenoptera. In: Brandão, C.R.F.; Cancello E.M. (Eds.). Biodiversidade do Estado de São Paulo, Brasil: síntesedo conhecimento ao final doséculoXX. Volume 5: invertebrados terrestres, São Paulo: FAPESP, 1999. Cap. 15, p.141-146.

DAILY, G.C; EHRLICH, P.H. Preservation of biodiversity in small rainforest patches: rapid evaluations using butterfly trapping. Biodiversity and Conservation , v.4, p.35-55, 1995.

ERFTEMEIJER, P.L.A. A new technique for rapid assessment of mangrove degradation: a case study of shrimp farm encroachment in Thailand. Trees-Structureand Function, v.16, p.204-208, 2002.

Fernández, F.; Siarkey, M. (Eds.) Introducción a los H ymen optera dela Región N eotropical . Bogotá: Sociedad Colombiana de Entomologia y Universidad Nacional de Colombia, 2006. 893p.

Grissell, E.E.;SChauff, M.E. (Eds.) A handbook of thefamilies of $\mathrm{N}$ eartic $\mathrm{C}$ halcidoidea (H ymenoptera). 2.ed. Washington:TheEntomological Society of Washington, 1997. 87p.

Hanson, P.E.; Gauld, I.D. (Eds.) The Hymenoptera of Costa Rica. Oxford: Oxford University Press, 1995. 893p.

HeRZOG, S.K.; KeSSLER, M.; CAHILL, T.M. Estimating species richness of tropical bird communities from rapid assessment data. Auk, Laurence, v.119, p.749-769, 2002.

IDAF - Instituto de Defesa A gropecuária e Florestal do Espírito Santo (ES). Plano de M anejo do ParqueE stadual da Pedra A zul. Vitória: Cepemar, 2004. 595p. (Relatório Técnico: RT 199-04). Disponível em: 丸ttp:/ / www.idaf.es.gov.br/ Pages/ wfParquePedra Azul. aspx>A cesso em: 29 ago. 2006.

LASALLE, J.; GAULD, I.D. Hymenoptera and biodiversity. Wallingford: C.A.B. International, 1993. 348p.

Mrs Estudos Ambientais. Plano de manejo do Parque Estadual do Forno Grande. Brasília: MRS ESTUDOS AMBIENTAIS, 2000a. 300p.

Mrs Estudos Ambientais. Plano de manejo do Parque Estadual da Cachoeira da Fumaça. Brasília: MRS ESTUDOS AMBIENTAIS, 2000b. 400p.

Oliver, I.; BeAtTIE, A.J. A possible method for the rapid assessment of biodiversity. Conservation Biology, Boston, v.7, p.562-568, 1993.

OıMı, M. A revision of the world Sclerogibbidae (Hymenoptera Chrysidoidea). Frústula Entomologia, Pisa, n. S., v.26-28, p.46-193, 2005.

Perioto, N.W.; LARA. R.I.R. Himenópteros parasitóides (Insecta: Hymenoptera) da Mata Atlântica. I. Parque Estadual da Serra do Mar, Ubatuba, SP, Brasil. A rquivos do Instituto Biológico, São Paulo, v.70, n.4, p.441-445, 2003. 
Perioto, N.W.; Lara, R.I.R.;Selegatto, A.Himenópteros parasitóides da Mata Atlântica. II. Núcleo Grajaúna, Rio Verde da Estação Ecológica JuréiaItatins, Iguape, SP, Brasil. A rquivos do Instituto Biológico, São Paulo, v.72, n.1, p.81-85, 2005. Disponível em: 〈http:/ / www. biologico.sp.gov.br/ ARQUIVOS/ V72 1/ perioto2. PDF>. A cesso: 29 ago. 2006.

Simonson, S.E.; Opler, P.A.; Stohlgren, T.J.; Chong, G.W. Rapid assessment of butterfly diversity in amontane landscape. Biodiversity and Conservation , v.10, p.13691386, 2001.
SMITH, W.; M EREDITH, T.C.; OHNS, T. Exploring methods for rapid assessment of woody vegetation in the Batemi Valley, North-central Tanzania. Biodiversity and Conservation , v.8, p.447-470, 1999.

Stohlgren, T.J.; Chong, G.W.; Kalkhan , M.A.; Schell, L.D. Rapid assessment of plant diversity patterns: a methodology for landscapes. Environmental M onitoring and A ssessment, v.48, p.25-43, 1997.

Recebido em 19/ 9/ 06 Aceito em 25/ 5/ 07 